\title{
Influência da razão Al/argila no processo de pilarização de esmectita
}

\section{(Influence of the Al/clay ratio on the pillaring process of smectite)}

\author{
D. L. Guerra', V. P. Lemos ${ }^{1}$, R. S. Angélica ${ }^{1}$, C. Airoldi ${ }^{2}$ \\ ${ }^{1}$ Centro de Geociências, Universidade Federal do Pará -UFPA, Belém, PA 66075-110 \\ ${ }^{2}$ Instituto de Química, Universidade Estadual de Campinas - UNICAMP \\ C.P. 6154, Campinas, SP 13083-970 \\ dlguerra@ufpa.br
}

\begin{abstract}
Resumo
As amostras de esmectita do estado do Pará, Amazônia, Brasil foram caracterizadas utilizando XRD, ${ }^{27}$ Al MAS NMR, FTIR e análise textural por curvas isotérmicas de adsorção-dessorção de nitrogênio. Na produção das argilas pilarizadas foi usado como íon intercalante, o $\mathrm{Al}_{13}$ (o íon de keggin), obtido pela reação das soluções $\mathrm{AlC}_{13} 6 \mathrm{H}_{2} \mathrm{O} / \mathrm{NaOH}$, com razão molar $\mathrm{OH} / \mathrm{Al}=2$. Foram adotadas as proporções (Al/Argila): 5, 10, 20 e $25 \mathrm{meq} / \mathrm{g}$ de argila, com intercalação em $25^{\circ} \mathrm{C}$, durante as $3 \mathrm{~h}$ e calcinada a $450{ }^{\circ} \mathrm{C}$ (temperatura adequada da calcinação). $\mathrm{O}$ resultado mostrou que o processo de pilarização aumentou o espaçamento basal da argila natural de 14,02 para 18,84 ̊̊ e a área superficial de 44,30 para 198,03 $\mathrm{m}^{2} / \mathrm{g}$ (Al/Argila=25meq/g de argila, $\left.\mathrm{pH}=4\right)$. $\mathrm{O}$ material preparado com relação $\mathrm{Al} / \mathrm{Argila}=25 \mathrm{meq} / \mathrm{g}$ de argila mostrou a incorporação máxima de $\mathrm{Al}$. A estabilidade térmica da argila natural foi melhorada pelo procedimento de pilarização.
\end{abstract}

Palavras-chave: argila pilarizada, $\mathrm{Al}_{13}$, esmectita.

\begin{abstract}
Smectite-type clays from the state of Pará, Brazil, were characterized by XRD, ${ }^{27}$ Al MAS NMR, FTIR and textural analysis by nitrogen adsorpition-desorption isotherms. In the production of pillared clays, the $\mathrm{Al}_{13}$ ion (keggin's ion) was used. This ion was obtained by the reaction of $\mathrm{AlC} \mathrm{C}_{13} 6 \mathrm{H}_{2} \mathrm{O} / \mathrm{NaOH}$ solutions with $\mathrm{OH} / \mathrm{Al}=2$ molar ratio and with $\mathrm{Al} /$ clay ratios of 5, 10, 15, 20, 25 meq/g of clay, intercalated at $25^{\circ} \mathrm{C}$ during $3 \mathrm{~h}$ and calcined at $450^{\circ} \mathrm{C}$ (adequate temperature for calcination). The results showed that the pillarization process increased the basal spaces of the natural clay from 14.02 to $18.84 \AA$ and the surface area from 44.30 to 198.03 $\mathrm{m}^{2} / \mathrm{g}(\mathrm{Al} / \mathrm{g}$ of clay $=25 \mathrm{meq} / \mathrm{g}$ of clay, $\mathrm{pH}=4)$. Materials prepared with different Al/g clay ratio showed the maximum Al incorporation for Al/g of clay $=25 \mathrm{meq} / \mathrm{g}$ of clay. The thermal stability of the natural clay was improved by the pillaring procedure.
\end{abstract}

Keywords: pillared clay, $\mathrm{Al}_{13}$, smectite.

\section{INTRODUÇÃO}

As argilas são utilizadas pelo homem nas mais diversas aplicações como, na antiguidade, na produção de utensílios domésticos e adornos de barro. As argilas são elementos componentes de uma grande parte de solos, podem ser encontradas no estado puro em depósitos minerais, em seu ambiente de formação e em ambientes naturais. A argila é responsável por uma série de processos considerados naturais, atuando como catalisadores, como por exemplo, em transformações químicas em solos e formação de petróleo. Minerais argilosos do grupo da caulinita, palikoskita e sepiolita ativados, têm amplo aspecto de aplicação em meio industrial.

As esmectitas possuem importante característica que é a possibilidade de expansão da distância interlamelar basal $\left(\mathrm{d}_{001}\right)$ que é a espessura da região situada entre as lamelas denominada de espaço interlamelar. Este espaço se expande quando é introduzido um íon de raio iônico podendo atingir até $40 \AA \AA$, no caso das esmectitas. Outro processo é o inchamento (swelling), provocado pelo equilíbrio de ionização entre os cátions adsorvidos e as superfícies das partículas que ocorre quando a argila é submetida a meio aquoso.

$$
\mathrm{M}-\text { Argila } \leftrightarrow \mathrm{M}^{+}+\text {Argila }
$$

As esmectitas quando ativadas através de tratamentos térmicos ou ácidos, são utilizadas nos mais diversos segmentos industriais como na indústria de óleos vegetais como descolorantes removendo substancias orgânicas, como caroteno, carotenóides e clorofila, atuando de maneira similar nas vinícolas [1].

Em 1955 foi criada uma nova classe de materiais porosos, as chamadas argilas pilarizadas. As pesquisas se intensificaram a partir de $1973 \mathrm{com}$ a crise mundial do petróleo, criando materiais porosos que viriam a ser utilizados 
no craqueamento de petróleo. Surge assim o processo de pilarização $[2,3]$. O processo de pilarização consiste na troca iônica entre íons presentes nos espaços interlamelares $\left(\mathrm{Na}^{+}\right.$e $\left.\mathrm{Ca}^{++}\right)$de uma esmectita com polihidroxicátions, originando preliminarmente uma argila intercalada, ou seja, a esmectita com o polihidroxicátion em sua estrutura na região interlamelar $[4,5]$. Através da calcinação, em temperaturas variadas $\left(300,450,600\right.$ e $\left.700{ }^{\circ} \mathrm{C}\right)$, é produzida a desidroxilação do íon intercalante resultando óxidos com estrutura rígida que recebem o nome de pilar (Fig. 1). Os pilares funcionam como sustentadores da estrutura esmectítica quando esta é submetida a altas temperaturas, além disto, é conferido ao sistema maior caráter ácido, devido aos grupos funcionais formados na parte externa dos pilares e da ligação pilar lamela que também produz sítios ácidos [6,7].

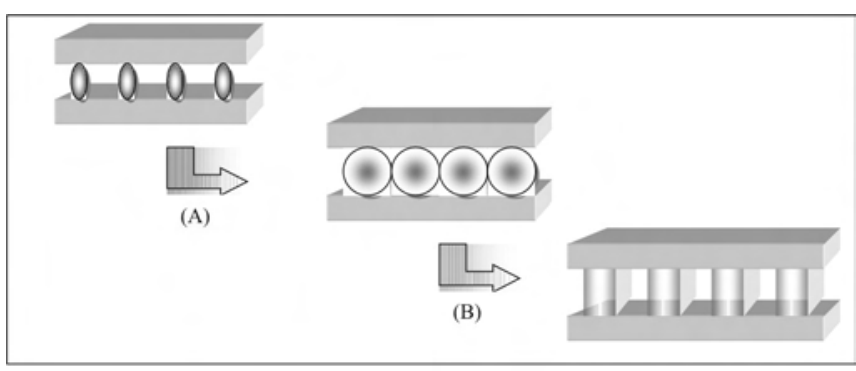

Figura1: Representação esquemática do processo de pilarização [7].

[Figure1: Schematic representation of the process of pilarization [7].]

O objetivo do processo de pilarização é conferir microporosidade ao sistema, alterando as suas propriedades físico-químicas, criando materiais contendo poros de dimensões complementares aos da zeólitas entre 7 e $20 \AA$ [8,9]. As argilas do grupo da esmectita são bastante utilizadas no processo de pilarização, entre elas destaca-se a montmorilonita com capacidade de troca catiônica (CTC) entre 40 a 150 meq/100 g de argila [10, 11].

As argilas pilarizadas são amplamente utilizadas como suporte de catalisadores e como adsorventes em grande número reações como na alquilação de tolueno com metanol e no craqueamento do petróleo[12]. Uma significativa variedade de polihidrocátions (Al, Fe, Zr, Cr, Ti e Ga) tem sido utilizada na preparação de argilas pilarizadas, entre os mais utilizados está o íon de Keggin $\left(\left[\mathrm{Al}_{13} \mathrm{O}_{4}(\mathrm{OH})_{24}\left(\mathrm{H}_{2} \mathrm{O}\right)_{12}\right]^{+7}\right)$, conhecido como $\mathrm{Al}_{13}$ [7].

Os parâmetros que têm sido avaliados na pilarização de esmectitas com $\mathrm{Al}_{13}$ são: A razão molar $\mathrm{OH} / \mathrm{Al}$, tempo de envelhecimento das soluções pilarizantes, $\mathrm{pH}$ e temperatura de calcinação [7-9], outro parâmetro de grande importância é a relação Al/ Argila. Estudos anteriores envolvendo a variação desta relação no desenvolvimento do processo de pilarização demonstraram que houve a formação de espécies monoméricas, poliméricas e o íon de Keggin [3, 7, 8].

No presente trabalho foi analisado a eficiência do agente pilarizante (íon de keggin) em suspensões aquosas (em temperatura ambiente) sob as relações 5, 10, 15, 20, 25 meq de Al/g de argila, no processo de pilarização de esmectita. Os demais parâmetros como composição química, temperatura de calcinação, tempo de envelhecimento etc., foram conservados em todos os experimentos.

\section{MATERIAIS E MÉTODOS}

\section{Material precursor}

A amostra natural de esmectita empregada pertence a um perfil laterítico, proveniente da Serra de Maicuru, no Município de Monte Alegre, Pará, Brasil, com capacidade de troca catiônica (CTC) igual a $58 \mathrm{meq} / 100 \mathrm{~g}$ de argila, obtido por acetato de amônio $(2 \mathrm{~N}, \mathrm{pH}=8) \mathrm{d}(001)=14,02$ Å. A amostra apresentou traços de caolinita e fração $\leq 2$, quando separada por sedimentação. Com fórmula estrutural: $\left(\mathrm{Si}_{2,54} \mathrm{Al}_{0,41}\right)^{\mathrm{IV}}\left(\mathrm{Al}_{2,48} \mathrm{Fe}_{079} \mathrm{Mg}_{0,06} \mathrm{Ti}_{0}{ }^{774}\right)^{\mathrm{VI}}\left(\mathrm{Ca}_{0,29} \mathrm{Na}_{0,21} \mathrm{~K}_{0,25}\right) \mathrm{O}_{20}(\mathrm{OH})_{4}$.

\section{Preparação da solução pilarizante de Al}

As soluções aquosas foram preparadas nas concentrações 5, 10, 15, 20, 25 meq/g de argila, com solução pilarizantes preparadas com $\mathrm{AlCl}_{3} \cdot 6 \mathrm{H}_{2} \mathrm{O}$ e $\mathrm{NaOH}$ na razão molar $\mathrm{OH} / \mathrm{Al}$ $=2$. As suspensões aquosas sob as condições meq $\mathrm{Al} / \mathrm{g}$ de argila $=5,10,15,20,25$ foram preparadas em temperatura de $25{ }^{\circ} \mathrm{C}$ sob agitação magnética por um período de $3 \mathrm{~h} \mathrm{e}$ envelhecidas por um período de 4 dias. Após este tempo de envelhecimento as suspensões apresentaram $\mathrm{pH}$ que variou de 4 a 4,5 .

\section{Processo de pilarização}

As suspensões aquosas foram misturadas com a solução pilarizante e agitadas por um período de $3 \mathrm{~h}$ em temperatura ambiente (processo de intercalação). Após a intercalação as amostras foram calcinadas em temperatura de $450^{\circ} \mathrm{C}$ e conservadas em dessecador.

\section{Técnicas de caracterização}

Para a difração de raios $\mathrm{X}$ (DRX) foi utilizado o método do pó para uma amostra sem orientação preferencial, em difratômetro Philips PW1050 controlado por um sistema PW 3710, com radiação $\mathrm{Cuk}_{\alpha}(1,54060$ $\AA$ ), monocromatizador e gerador de tensão e corrente para $45 \mathrm{kV}$ e $35 \mathrm{~mA}$, e varredura de $1 \%$ min.

As amostras foram analisadas por espectrometria de absorção atômica ( $\mathrm{Na}, \mathrm{K}, \mathrm{Ca}, \mathrm{Mg}, \mathrm{Al})$, espectrofotometria na região visível $(\mathrm{Si}, \mathrm{Fe}, \mathrm{Ti})$ sendo o $\mathrm{Si}$ pelo método azul de molibdênio, Fe sob a forma do complexo ortofenantrolina ferrosa e Ti como peróxido complexo.

Os espectros de ressonância magnética nuclear $\left(\mathrm{RMN},{ }^{27} \mathrm{Al}\right)$ das argilas pilarizadas, foram obtidos em espectrômetro Gemini-300 Varian e AC 300/P Bruker. 
Com um comprimento de pulso $\mathrm{PW}(\pi / 2)=0,7 \mu \mathrm{s}$, tempo de aquisição $\mathrm{AQ}=0,050 \mathrm{~s}$, intervalo entre pulsos $\mathrm{DI}=$ $0,3 \mathrm{~s}$, número de transientes $\mathrm{NT}=500 \mathrm{e}$ as referências foram $\mathrm{ALCl}_{3} \cdot 6 \mathrm{H} 2 \mathrm{O}(\delta=0 \mathrm{ppm})$. Foi utilizada uma sonda multinuclear para tubos de $5 \mathrm{~m}$ de diâmetro externo contendo água deuterada, empregando solução de $\mathrm{AlCl}_{3}$ $(1,0 \mathrm{M})$ como referência.

Para obtenção dos espectros de FTIR, foram utilizados $0,200 \mathrm{~g}$ de $\mathrm{KBr}$ e $0,0013 \mathrm{~g}$ de amostra pulverizada, foi confeccionada uma partilha pelo método de prensagem com 7 ton utilizando vácuo, objetivando a remoção da umidade e auxiliando a prensagem e para a solução pilarizante, utilizou-se uma célula apropriada para análise de líquidos, em um espectrômetro de absorção molecular na região IV com transformada de Fourier, Perkin Elmer FT-IR 1760 X.

A determinação da área específica (AE), volume total dos poros (VTP) e diâmetro médio dos poros (DMP) foi obtida a partir de 0,249 g de cada amostra. As amostras foram aquecidas a $150{ }^{\circ} \mathrm{C}$ durante $1 \mathrm{~h}$. Após o resfriamento, foram colocadas em contato com nitrogênio líquido em um analisador de adsorção gasosa Quantachrome/nova-1200/5. Através das análises foram obtidas as respectivas isotermas de adsorção-desorção. A partir dos dados das isotermas foram calculados os valores de AE e VP. A área especifica foi calculada pelo método BET (Brunauer-Emmett-Teller) e o volume total de poros, calculado a pressão parcial $\left(\mathrm{p} / \mathrm{p}_{0}\right)$ de 0,998955 pelo método BJH (Barrett-Joyner-Halenda).

Para a obtenção das curvas de análise termogravimétrica (ATG) foi utilizado um aparelho termoanalisador Stanton Redcroft. A análise foi feita de 25 a $1100{ }^{\circ} \mathrm{C}$ com uma taxa de aquecimento de $20{ }^{\circ} \mathrm{C} / \mathrm{min}$.

Para as análises de microscopia eletrônica de varredura (MEV), as amostras foram preparadas em laminas orientadas a $25^{\circ} \mathrm{C}$ e metalizadas com ouro. Foram realizadas varreduras em todas as lâminas para a obtenção de imagens e análises químicas pelo método EDS. O instrumento utilizado foi LEO-ZEISS, $430 \mathrm{Vp}$, em condições de análise utilizando imagens secundárias obtidas a $20 \mathrm{kV}$, com distância de trabalho de $11 \mathrm{~mm}$.

\section{RESULTADOS E DISCUSSÃO}

\section{Difração de raios $X$}

Através dos dados de DRX (Tabela I e Fig. 2) foi observado que os valores de $d(\AA)$ das reflexões $\mathrm{d}(001)$ tendem a aumentar gradualmente entre 5 a $10 \mathrm{meq} \mathrm{Al} / \mathrm{g}$ de argila e valores com um pequeno aumento no intervalo de 15 a $25 \mathrm{meq} \mathrm{Al} / \mathrm{g}$ de argila. Este comportamento sugere que a preparação de $\mathrm{Al}_{2} \mathrm{O}_{3}$ nas esmectitas pilarizadas tenha se estabilizado neste intervalo.
Tabela I - Valores de d(001) para a argila natural e argila pilarizadas.

[Table I - Values of d(001) for the natural clay and pillared clays]

\begin{tabular}{cc}
\hline Amostras Pilarizadas* $^{*}$ & $\mathrm{~d}(\AA)$ \\
\hline Natural & 14,02 \\
PILC $_{5}$ & 16,74 \\
PILC $_{10}$ & 17,60 \\
PILC $_{15}$ & 18,10 \\
PILC $_{20}$ & 18,45 \\
PILC $_{25}$ & 18,84 \\
\hline
\end{tabular}

*Temperatura de calcinação igual a $450^{\circ} \mathrm{C}$.

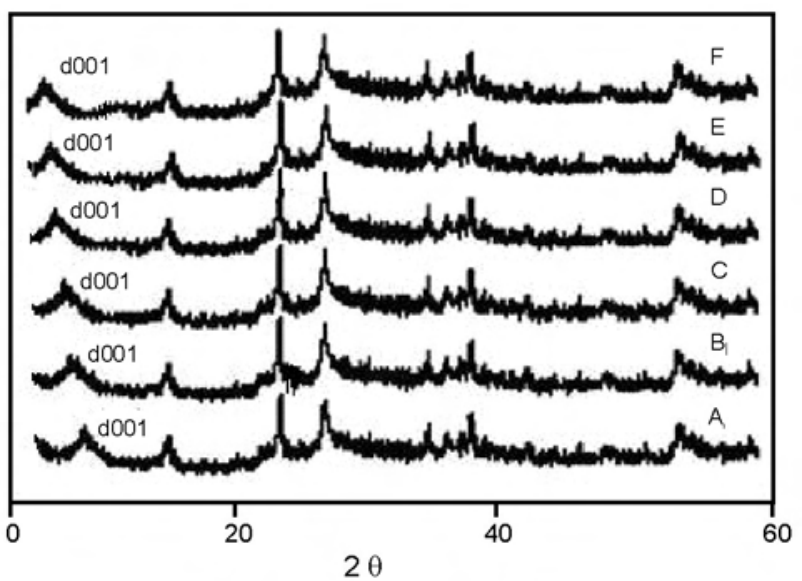

Figura 2: Difratogramas de raios X para: (A) Natural; (B)PILC ${ }_{5}$; (C)PILC 10 ; (D)PILC 15 ; (E)PILC 20 ; (D)PILC PI $_{20}$.

[Figure 2: X-ray diffraction patterns of: (A) Natural; (B)PILC $C_{5}$; (C)PILC ${ }_{10}$; (D)PILC ${ }_{15}$; (E)PILC 20 ; (D)PILC ${ }_{25}$ ].

\section{Análises químicas}

Com base nos dados das analises químicas antes e depois da pilarização (Tabela II) é possível verificar a quantidade de alumínio incorporada à estrutura da esmectita. Estes dados foram utilizados para o cálculo do número de pilares (NP).

Tabela II - Composição química das amostras natural e pilarizadas.

[Table II -chemical composition of the natural and pillared samples].

\begin{tabular}{ccccc}
\hline Amostras & $\% \mathrm{Al}_{2} \mathrm{O}_{3}$ & $\% \mathrm{CaO}$ & $\% \mathrm{Na}_{2} \mathrm{O}$ & $\% \mathrm{~K}_{2} \mathrm{O}$ \\
\hline Natural & 17,89 & 4,78 & 1,204 & 3,80 \\
PILC $_{5}$ & 26,90 & 2,70 & 1,050 & 2,15 \\
PILC $_{10}$ & 26,95 & 1,91 & 0,199 & 2,10 \\
PILC $_{15}$ & 28,09 & 1,81 & 0,199 & 2,01 \\
PILC $_{20}$ & 28,90 & 1,78 & 1,198 & 1,94 \\
PILC $_{25}$ & 28,94 & 1,74 & 0,178 & 1,90 \\
\hline
\end{tabular}




\section{Número de pilares}

O número de pilares é calculado com base no percentual de alumínio incorporado na estrutura da esmectita, mais especificamente na região interlamelar, Este valor é relacionado com o número de átomos de alumínio (Equação B) [3] envolvido no processo de pilarização (Fig. 3 e Tabela III). Foi verificado que o número de pilares alcançou valor mais expressivo em altas concentrações, este fato pode ser explicado pela maior produção de $\mathrm{Al}_{13}$, conseqüentemente haverá uma entrada maior do íon na estrutura da esmectita, formando maior número de pilares.

$2\left[\mathrm{Al}_{13} \mathrm{O}_{4}(\mathrm{OH})_{24}\left(\mathrm{H}_{2} \mathrm{O}\right)_{12}\right]^{+7} \rightarrow 13 \mathrm{Al}_{2} \mathrm{O}_{3}+41 \mathrm{H}_{2} \mathrm{O}+14 \mathrm{H}^{+}$

$\underline{\partial \mathrm{N}}=\left\{\eta\left[\left(\mathrm{z} \mathrm{M}_{\mathrm{z}}\right)+\left(\check{\mathrm{n}}_{\mathrm{n}}\right)\right\}^{-1} \partial \mathrm{C}_{\mathrm{Al}}\right.$

Onde $\mathrm{N}=$ número de pilares, $\mathrm{C}_{\mathrm{Al}}=$ Concentração de Alumínio, $\eta=$ número de átomos de alumínio, $\mathrm{z}=$ número de prótons, $\check{\mathrm{n}}$ = número de nêutrons, $\mathrm{M}_{\mathrm{z}}=$ massa do próton $\left(1,67252 \cdot 10^{-24} \mathrm{~g}\right)$, $\mathrm{M}_{\mathrm{n}}=$ massa do nêutron $\left(1,67483 \cdot 10^{-24} \mathrm{~g}\right)$

Tabela III - Percentual de $\mathrm{Al}_{2} \mathrm{O}_{3}$ incorporado e número de pilares na estrutura.

[Table III - Incorporated perceptual of $\mathrm{Al}_{2} \mathrm{O}_{3}$ and number pillars in the structure.]

\begin{tabular}{cccc}
\hline Amostras & $\% \mathrm{Al}_{2} \mathrm{O}_{3}$ & $\begin{array}{c}{\mathrm{O} \mathrm{Al}_{2} \mathrm{O}_{3}}_{\text {Incorporado }} \\
\text { (Calculado) }\end{array}$ & $\begin{array}{c}\text { Número de } \\
\text { Pilares } \\
- \text { NP/g de } \\
\text { argila } \\
(\mathrm{X} \mathrm{10})\end{array}$ \\
\hline Natural & 17,89 & - & - \\
IILC $_{5}$ & 26,90 & 20,01 & 4,522 \\
PILC $_{10}$ & 26,95 & 20,30 & 4,596 \\
PILC $_{15}$ & 28,09 & 22,90 & 5,184 \\
PILC $_{20}$ & 28,90 & 23,01 & 5,209 \\
PILC $_{25}$ & 28,94 & 24,20 & 5,478 \\
\hline
\end{tabular}

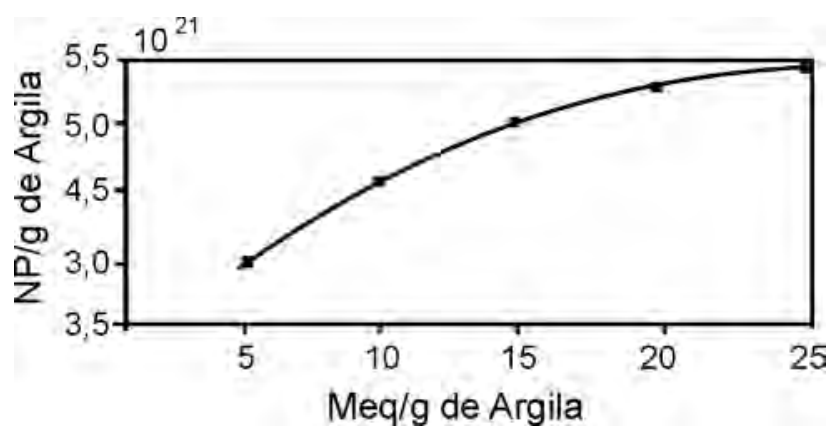

Figura 3: Quantidade de pilares na região interlamelar da esmectita em função da razão meqAl/g de argila.

[Figure 3: Quantity of pillars in the region interlayer of the esmectita in function of the meqAl/g of clay ratio].

\section{Análise por $R M N-M A S^{27} \mathrm{Al}$}

Na Fig. 4 estão apresentados os espectros de RMN-MAS de ${ }^{27} \mathrm{Al}$. Foram observados sinais na posição tetraédrica $(\delta=68 \mathrm{ppm})$ e alumínios na posição octaédrica $(\delta=0 \mathrm{ppm})$ com percentuais de 15 e $85 \%$ respectivamente, o que pode indicar uma possível deficiência de carga na camada tetraédrica, este fato favorece a presença de íons na região interlamelar. Também foi verificado um pequeno sinal na região de $58,7 \mathrm{ppm}$, que pode ser atribuído ao alumínio tetraédrico o que pode estar relacionado com a presença do íon de keggin $\left(\mathrm{Al}_{13}\right)$ na região interlamelar. Com a calcinação não foi observada mudança significativa nos espectros.

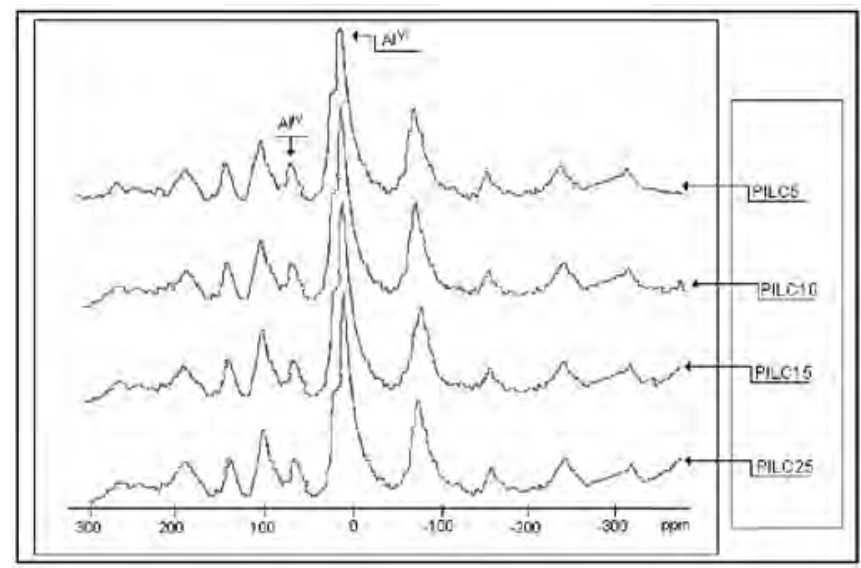

Figura 4: Espectros de RMN-MAS ${ }^{27} \mathrm{Al}$ das amostras pilarizadas com $\mathrm{Al}_{13}$. [Figure 4: Spectrum of ${ }^{27}$ Al MAS-NMR of the pillared samples with $\mathrm{Al}_{13}{ }_{13}$.

\section{Análise da solução pilarizante por FTIR}

Através do espectro de FTIR foi possível observar na solução pilarizante um pico de absorção na região de $663 \mathrm{~cm}^{-1}$ nas soluções em razão molar igual a 2 , este pico é atribuído à ligação Al-O em coordenada tetraédrica no centro do íon de Keggin. Este resultado comprova a existência do íon de Keggin em solução (Fig. 5).

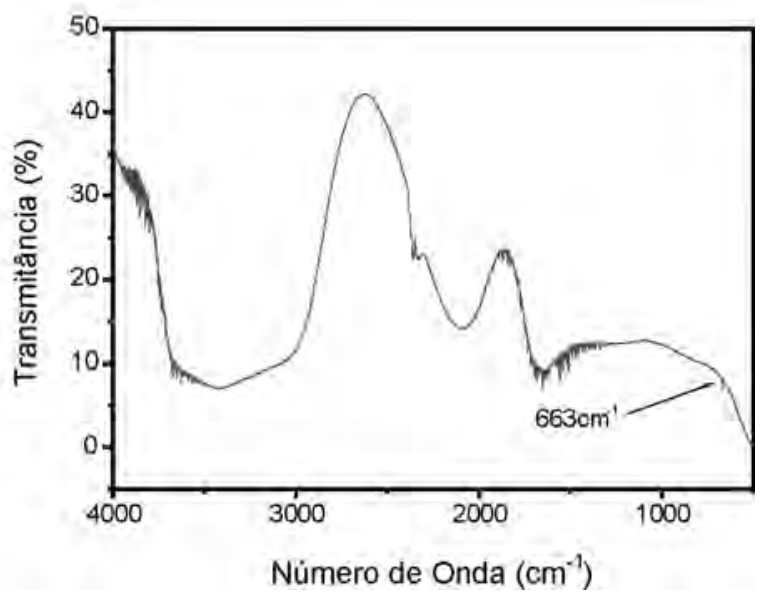

Figura 5: Espectro de FTIR da solução com $\mathrm{Al}_{13}$ ( razão molar=2). [Figure 5: FTIR spectrum of the solution with $\mathrm{Al}_{13}$ (molar ratio=2)]. 


\section{Análise das argilas pilarizadas por FTIR}

Com análise nos espectros fornecidos pelo FTIR, foi observado uma pequena banda de absorção na região de $667 \mathrm{~cm}^{-1}$ na esmectitas pilarizadas, banda semelhante aquela observada na análise da solução pilarizante, em todas as relações Al/Argila, na esmectita em estado natural esta banda não é observada, a formação desta banda é atribuída à ligação Al-O em coordenada tetraédrica no centro do pilar de alumínio, banda atribuída a esta ligação também foi encontrado na literatura [9] na região de $642 \mathrm{~cm}^{-1}$ (Fig. 6).

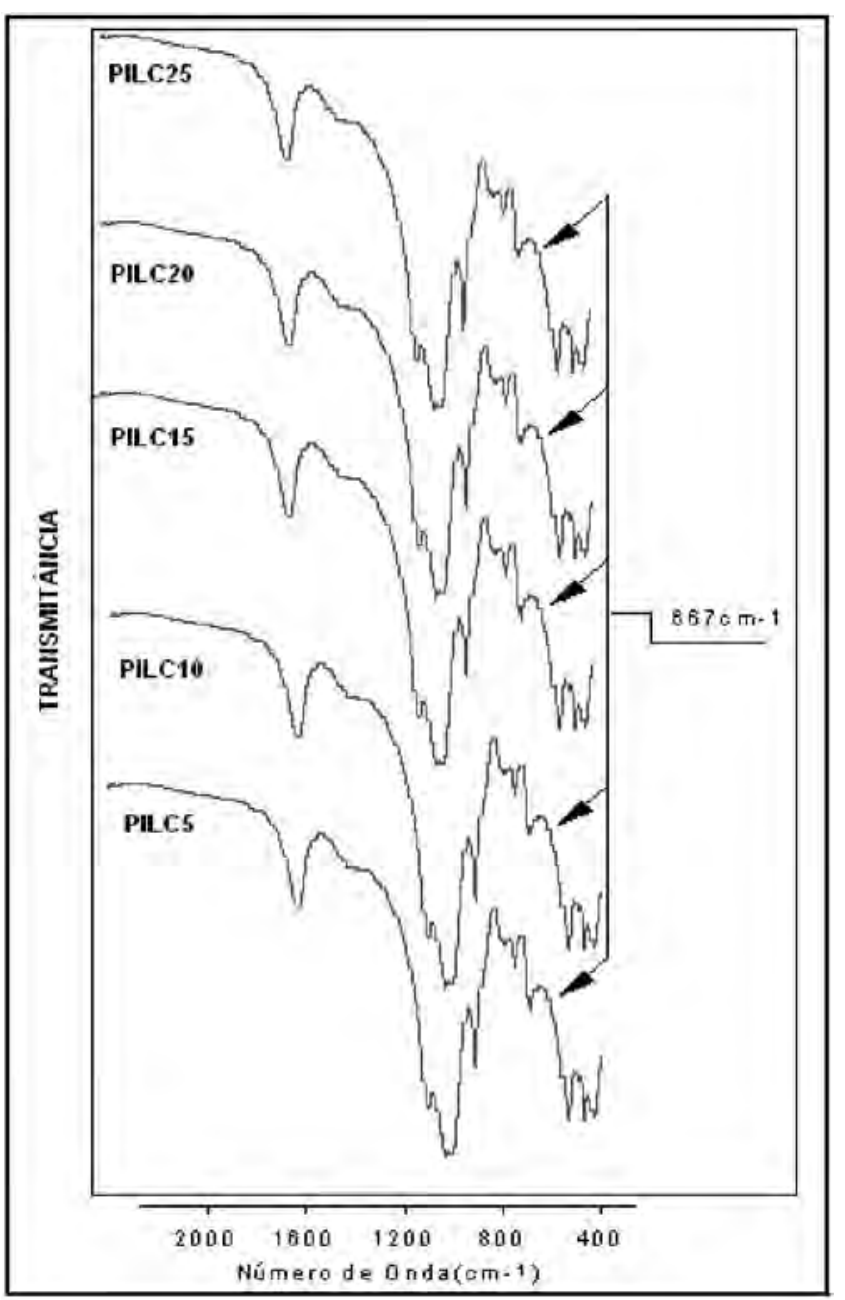

Figura 6: Análise de FTIR das argilas pilarizadas com $\mathrm{Al}_{13}$.

[Figure 6: FTIR analysis of the pillared clays with $\mathrm{Al}_{13}$.]

\section{Análise textural}

Na Tabela IV estão apresentados os resultados da análise textural: Área específica (AE); Volume total de poros (VTP); Diâmetro médio dos poros (DMP). Foi verificado que com a pilarização o valor da área específica cresceu atingindo valor máximo na relação $\mathrm{Al} /$ argila de $25 \mathrm{meq} / \mathrm{g}$ de argila. Analisando as isotermas de adsorção/desorção de $\mathrm{N}_{2}$, formadas para as amostras pilarizadas, é observado que elas pertencem a classificação do tipo IV, segundo BDDT (Brunauer, Deming, Deming e Teller) [7-9] (Fig. 7). Houve a formação de ciclo de histerese em altas pressões relativas, em todas as amostras pilarizadas, característica dos materiais mesoporosos, podendo conter microporos. Um ligeiro afastamento de curva é observado em baixas pressões. Este tipo de efeito está relacionado com fenômeno de expansão que é característico de materiais cujo grau de expansão do sólido, durante a adsorção, depende da rigidez deste sólido.

Análise termogravimétrica (ATG)

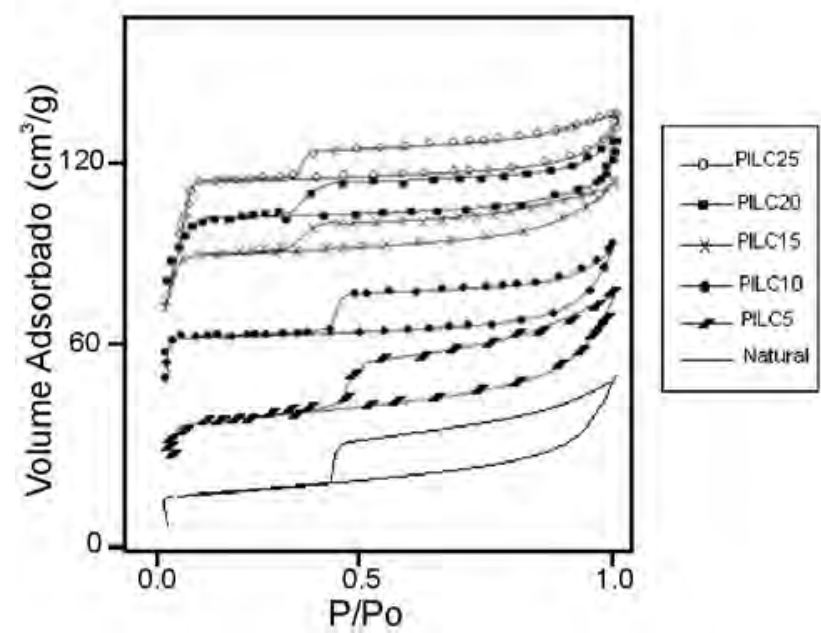

Figura 7: Isotermas de adsorção-desorção das amostras pilarizadas com $\mathrm{Al}_{13}$.

[Figure 7: Isotherms of desorption-adsorption of the pillared samples with $\mathrm{Al}_{13}$ ].

Tabela IV - Resultados da análise textural da amostra natural e amostras pilarizadas.

[Table IV - Results of textural analysis of the natural sample and pillared sample].

\begin{tabular}{cccc}
\hline Amostras & $\begin{array}{c}\mathrm{AE} \\
\left(\mathrm{m}^{2} / \mathrm{g}\right)\end{array}$ & $\begin{array}{c}\mathrm{VTP} \\
\left(\mathrm{cm}^{3} / \mathrm{g}\right)\end{array}$ & $\begin{array}{c}\mathrm{DMP} \\
(\AA)\end{array}$ \\
\hline Natural & 44,30 & 0,25 & 45,09 \\
PILC & 129,30 & 0,28 & 130,01 \\
PILC $_{10}$ & 138,03 & 0,30 & 140,87 \\
PILC $_{15}$ & 141,90 & 0,33 & 145,89 \\
PILC $_{20}$ & 155,90 & 0,39 & 157,40 \\
PILC $_{25}$ & 198,03 & 0,47 & 176,30 \\
\hline
\end{tabular}

Através das curvas termogravimétricas foi verificado que a perda de água é maior nas argilas pilarizadas nas relações 20 e 25 meq/ g de argila, fato atribuído à existência de maior número de pilares na região interlamelar, é observado também uma perda maior de água nas amostras pilarizadas nas mesmas relações, em temperatura acima 
de $600{ }^{\circ} \mathrm{C}$, com sinal de desidroxilação considerável possivelmente explicado pela colapsação total da estrutura esmectítica pilarizada (Fig. 8). É também observado que argilas pilarizadas em maior relação possuem maior resistência a altas temperaturas, fato também atribuído a densidade dos pilares. Com o aumento da temperatura ocorre a conversão do oligômero de $\mathrm{Al} \mathrm{a} \mathrm{Al}_{2} \mathrm{O}_{3}$ com perda de água segundo a Equação B [7, 8].

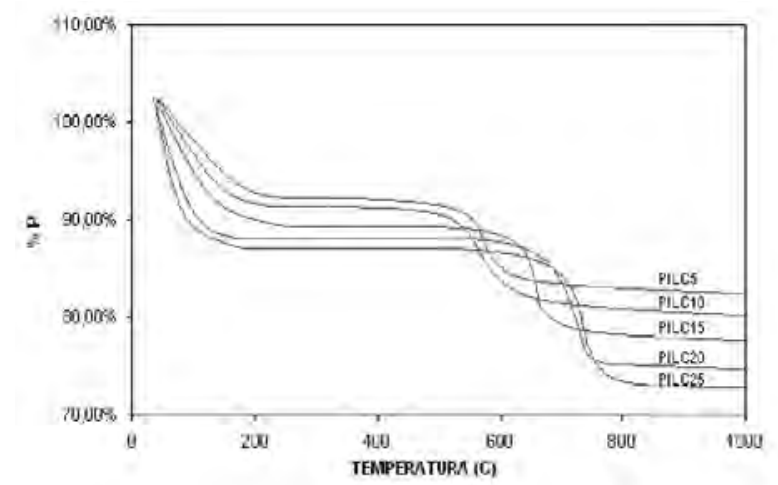

Figura 8: Curvas de ATG de argilas pilarizadas com $\mathrm{Al}_{13}$. [Figure 8: TGA curves of pillared clays with $\mathrm{Al}_{13}$.]

\section{Microscopia Eletrônica de Varredura (MEV)}

As imagens do MEV obtidas para as amostras: natural e pilarizadas foram idênticas; foi observado (Fig. 9) que as amostras se apresentam de maneira foliada. Na extremidade das "folhas" verificase a presença de ilita em baixa concentração. Os resultados de EDS (Figs. 10, 11 e Tabela V) mostram o aumento dos teores de $\mathrm{Al}_{2} \mathrm{O}_{3}$ na amostra pilarizada e decréscimo nos teores de $\mathrm{Fe}_{2} \mathrm{O}_{3}, \mathrm{CaO}, \mathrm{MgO}, \mathrm{TiO}_{2}$ e $\mathrm{Na}_{2} \mathrm{O}$. Os valores fornecidos por este método são bastante próximos aos obtidos através de outros métodos de analises químicas, a concentração de ilita nas extremidades das folhas foram detectadas por analise pontual semiquantitativa pelo método EDS.

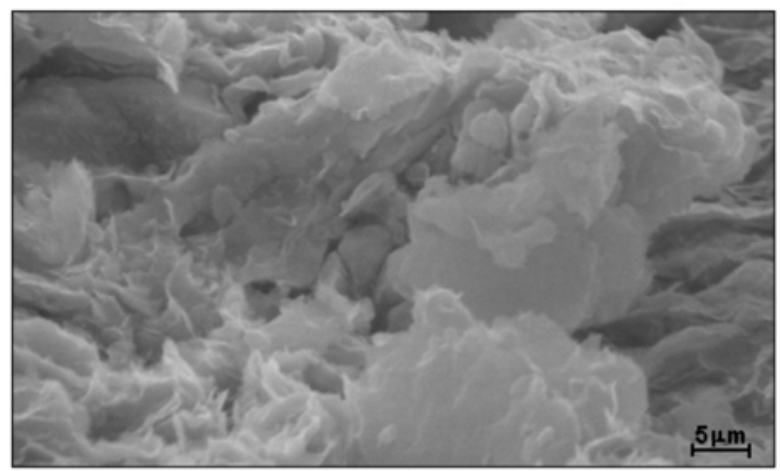

Figura 9: Micrografia obtida em microscópio eletrônico de varredura da esmectita natural.

[Figure 9: Scanning electron microscopy micrograph of natural smectite].

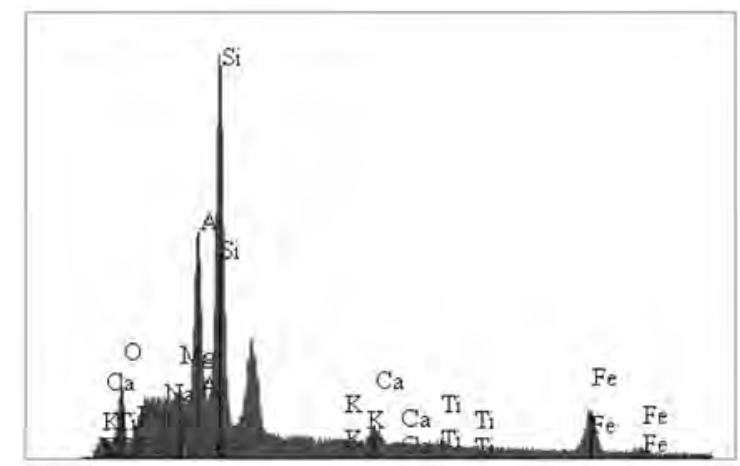

Figura 10: Análise EDS da amostra natural. [Figure 10: EDS analysis of the natural sample].

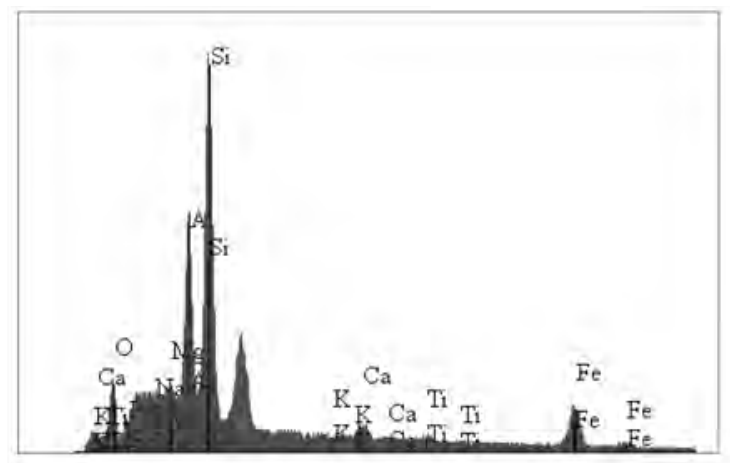

Figura11. Análise EDS da amostra pilarizada (PILC $\left.{ }_{0,5}\right)$. [Figure 11: EDS analysis of the pillared sample (PILC ${ }_{0.5}$.].

Tabela V - Resultados das análises pelo método EDS das esmectitas natural e pilarizadas. [Table V - EDS analysis of natural and pillarized smectites.]

\begin{tabular}{ccccccccc}
\hline Amostras & $\% \mathrm{SiO}_{2}$ & $\% \mathrm{Al}_{2} \mathrm{O}_{3}$ & $\% \mathrm{Fe}_{2} \mathrm{O}_{3}$ & $\% \mathrm{TiO}_{2}$ & $\% \mathrm{Na}_{2} \mathrm{O}$ & $\% \mathrm{~K}_{2} \mathrm{O}$ & $\% \mathrm{MgO}$ & $\% \mathrm{CaO}$ \\
\hline Natural & 40,01 & 18,18 & 19,52 & 2,21 & 1,90 & 3,70 & 8,68 & 5,80 \\
PILC $_{5}$ & 39,71 & 26,01 & 17,97 & 2,18 & 1,75 & 2,19 & 8,19 & 2,00 \\
PILC $_{10}$ & 39,70 & 27,03 & 17,96 & 2,17 & 0,99 & 2,18 & 7,98 & 1,99 \\
ILCC $_{15}$ & 39,61 & 28,88 & 17,63 & 2,00 & 0,80 & 2,15 & 7,39 & 1,54 \\
PILC $_{20}$ & 39,60 & 29,94 & 17,28 & 1,97 & 0,76 & 1,95 & 7,10 & 1,40 \\
PILC $_{25}$ & 39,62 & 29,97 & 17,27 & 1,96 & 0,75 & 1,94 & 7,09 & 1,40 \\
\hline
\end{tabular}




\section{CONCLUSÕES}

Os resultados obtidos neste estudo indicaram que o intervalo da relação meq Al/g de argila $=5-25 \mathrm{meqAl} / \mathrm{g}$ de argila obteve satisfatório desempenho na pilarização da amostra de esmectita $\left(\mathrm{Si}_{2.54} \mathrm{Al}_{0.41}\right)^{\mathrm{IV}}\left(\mathrm{Al}_{2,48} \mathrm{Fe}_{079} \mathrm{Mg}_{0,06} \mathrm{Ti}_{0}{ }^{7} 4\right)$ ${ }^{V I}\left(\mathrm{Ca}_{0,29} \mathrm{Na}_{0,21} \mathrm{~K}_{0,25}\right) \mathrm{O}_{20}(\mathrm{OH})_{4}$ de Maicuru-Pará. Os maiores valores da reflexão $\mathrm{d}_{001}$ foram obtidos para as frações da amostra pilarizada com 15 a $25 \mathrm{meqAl} / \mathrm{g}$ de argila sendo os valores de $d(\AA)=18,10$ a 18,84 , respectivamente, com a calcinação há um decréscimo nos valores de $\mathrm{d}_{001}$ obtidos com a intercalação, tal fato é atribuído a desidroxilação dos pilares. Outro fato relevante é a estabilização do valor de $\mathrm{d}_{001}$ no intervalo 15 a $25 \mathrm{meq} / \mathrm{g}$ de argila, este fato é explicado pela influência da etapa final no processo de substituição iônica, ou seja, os sítios catiônicos trocavéis presentes na região interlamelar da argila natural estariam em grande número, substituídos pelo íon de alumínio. Com a estrutura da argila alcançando a neutralidade, o valor de $\mathrm{d}_{001}$ apresenta pequena variação, pois a argila diminui significativamente $o$ seu poder de troca catiônica, não mais reagindo com a solução pilarizante em condições de saturação elevada, esta pequena variação de $\mathrm{d}_{001}$ também pode ter influência da calcinação como, por exemplo, a desidroxilação prematura de um pilar mal formado ou mal posicionado na região interlamelar.

Foi verificado um aumento significativo na concentração de $\mathrm{Al}_{2} \mathrm{O}_{3}$ e no número de pilares sob condições semelhantes no processo de pilarização, com as analises de FTIR foi

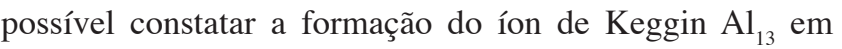
solução, através da banda na região $663 \mathrm{~cm}^{-1}$ e nas argilas pilarizadas em $667 \mathrm{~cm}^{-1}$ comprovando o estudo desenvolvido por Kloprogge et al. (2002) [9]. As analises de RMN-MAS ${ }^{27} \mathrm{Al}$ forneceram informações importantes sobre uma possível deficiência de carga na camada tetraédrica, atribuídas a substituição de silício por alumínio [3] favorecendo com este fato o processo de troca catiônica entre a região interlamelar da argila e o meio saturado (solução pilarizante).

O aumento da área específica está relacionado com o número de pilares presentes na região interlamelar, ou seja, o elevado maior número de pilares diminui de maneira significativa a probabilidade da ocorrência de concentração excessiva de pilares nas bordas das lamelas, este acontecimento é bastante comum, quando o método de pilarização é desenvolvido sem um agente espaçador, originando uma argila parcialmente pilarizada, fato que explica a má forma do pico $\mathrm{d}_{001}$, após a submissão a temperatura de calcinação, pois parte da estrutura é colapsada e parte é sustentada pelos pilares.

Há uma influência significativa da relação Al/g de argila no processo de pilarização e no material produzido, com importante efeito em suas propriedades físico-quimicas, havendo consequentemente comprometimento da eficácia deste material, na aplicação em reações catalíticas e em processos adsortivos.

\section{AGRADECIMENTOS}

Ao Centro de Geociências (CG-UFPA) e Instituto de Química (UNICAMP) com o seu corpo técnico e ao CNPq pelo suporte financeiro.

\section{REFERÊNCIAS}

[1] I. R. Diaz, D. Santos, Studies on the acid activation of Brazilian smectite clay, Química Nova 24 (2001) 315-353.

[2 ] J. F. Luna, U. Schuchardt, Química Nova 22 (1999) 104109.

[3] S. B. C. Pergher, A. Corma, V. Fornes, Química Nova. 22 (1999) 693-708.

[4] P. Cañizares, J. L. Valverde, M. R. Sun Kou, C. B. Molina, Microporous Mesoporous Mater. 29 (1999) 267-281.

[5] A. Bahamonde, F. Mohino, M. Rebollar, M. Yates, P. Avila, S. Mendioroz, Catalysis Today 69 (2001) 233-239.

[6] C. Volzone, Microporous Mesoporous Mater. 49 (2001) 197-202.

[7] D. J. L Guerra, V. P. Lemos, R.S. Angélica, C. Airoldi, Ciênc. Tecnol. Mater. 17 (2005) 75.

[8] J. Sterte, Pillared Clays, Catalysis Today 2 (1988) 219.

[9] J. T. Kloprogge, R. Evans, L. Hickey, R.L. Frost, Appl. Clay Sci. 20 (2002) 157-163.

[10] D. J. L Guerra, V. P Lemos, R. S. Angélica, E. F. Fernandes, M. L. Costa, Ecl. Quim. 30 (2005) 27.

[11] M. L. Occelli, R. M. Tindwa, Clays Clay Minerals. 32 (1983) 22-28.

[12] S. Moreno, E. Guitierrez, A. Alvarez, N. G. Papayannakos, G. Poncelet, Appl. Catal. 165 (1997) 103104.

[13] S. Q. M. Leite, R. A. S. San Gil, S. M. C. Menezes, Química Nova 23, 2 (200) 149-154.

(Rec. 17/08/2005, Rev. 16/11/2005, Ac. 12/05/2006.) 